\title{
Zukunftsfähigkeit gefährdet
}

VON JOSEF HILBERT,

MICHAELA EVANS UND

VIACHESLAV GALCHENKO

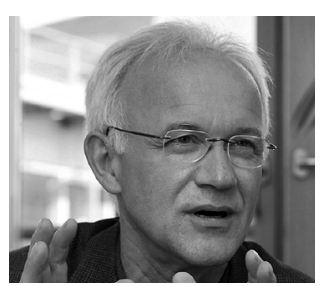

PD Dr. Josef Hilbert ist geschäftsführender Direktor des Instituts Arbeit und Technik (IAT) der Westfälischen Hochschule und Direktor des Forschungspunktes "Gesundheitswirtschaft und Lebensqualität«.

hilbert@iat.eu

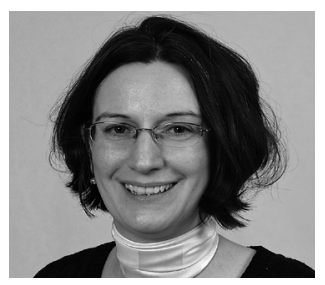

Michaela Evans ist seit 1999 wissenschaftliche Mitarbeiterin am Institut Arbeit und Technik (IAT) der Westfälischen Hochschule und Projektbereichsleiterin für das Themenfeld "Arbeit und Qualifizierung« im Forschungsschwerpunkt »Gesundheitswirtschaft und Lebensqualität«. evans@iat.eu

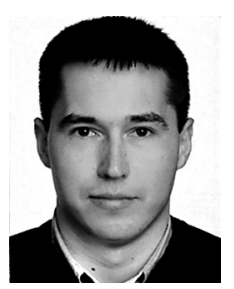

Viacheslav Galchenko ist seit 2010 die wissenschaftliche Hilfskraft am Institut Arbeit und Technik (IAT) der Westfälischen Hochschule im Forschungsschwerpunkt "Gesundheitswirtschaft und Lebensqualität« tätig. galchenko@iat.eu.

\author{
Der Einfluss von Lohn- und Arbeitsbedingungen auf \\ die Entwicklungschancen sozialer Dienstleistungen \\ untersuchte ein Studienprojekt in elf Ländern der \\ Europäischen Union. Das zentrale Ergebnis für \\ Deutschland: Die Zukunftsbranche Sozialwirtschaft \\ droht sich durch zersplitterte Repräsentations- und \\ Verhandlungsstrukturen selbst auszubremsen.
}

Die Generaldirektion Beschäftigung der Europäischen Union hat ein Netzwerk von Europäischen Interessenorganisationen der Sozialwirtschaft beauftragt, eine Expertise über die Zukunft des sozialen Dialogs in der Sozialwirtschaft durchzuführen.

Ein besonderes Augenmerk sollte dabei auf die Frage gelegt werden, ob und wie die Organisation der arbeitspolitischen Repräsentations- und Verhandlungsstrukturen der Arbeitgeberseite sich auf die Tarif- und Arbeitsbedingungen auswirkt. Unter Sozialwirtschaft wurden dabei die Altenhilfe, die Kinder- und Jugendhilfe sowie die Behindertenhilfe verstanden.

Die Studie sollte praxisfundiert und gestaltungsorientiert sein, was sich auch in ihrem Titel »Promoting Employers' Social Services Organisations in Social Dialogue « ausdrückt. Die Gesamtexpertise beruht auf Länderstudien, die in insgesamt elf Ländern der Europäischen Union durchgeführt wurden; in Deutschland lag die Federführung der Aktivitäten bei der Arbeiterwohlfahrt, die wissenschaftlichen Erhebungen und deren Interpretationen wurden vom Institut Arbeit und Technik (IAT) durchgeführt.

Hintergrund und Anlass für diese Studie war,

- dass offensichtlich in vielen europäischen Ländern die Lohn- und Arbeitsbedingungen in der Sozialwirtschaft schlechter sind als in vielen anderen Wirtschaftsbereichen,
- dass dies Zweifel aufkommen lässt, ob die Branche in Zukunft den wachsenden Herausforderungen - vor allem bei der Pflege Älterer und bei der Betreuung von Kindern - gerecht werden kann,

- dass deshalb überprüft werden muss, ob es sinnvoll ist, auf Ebene der Europäischen Union wie auch in den EU-Mitgliedsländern, einen sozialen Dialog zwischen Arbeitgeberorganisationen, Gewerkschaften und Politik über Wege zu mehr Zukunftsfähigkeit aufzubauen,

- wie und unter welchen Bedingungen sich die Arbeitgeberseite an einem solchen Austausch beteiligen könnte.

Die folgenden Ausführungen geben zunächst die zentralen Ergebnisse der deutschen Länderstudie wieder und unterbreiten dann Anregungen, welche Konsequenzen seitens der einschlägigen Wirtschaft und der Politik gezogen werden könnten und wo weitere Forschung notwendig erscheint.

\section{Methoden}

Die am Forschungsverbund beteiligten Teams hatten vereinbart, sich auf die Beantwortung eines gemeinsamen Fragekanons zu konzentrieren; seine wichtigsten Frageblöcke waren:

- Welche Bedeutung hat die Sozialwirtschaft für Wirtschaft und Arbeitsmarkt? 
- Welche Formen sozialen Dialogs und tariflicher Vereinbarungen existieren?

- Wie sind Arbeitgeber organisiert und wie beteiligen sie sich (auf welcher Ebene) am sozialen Dialog?

- Wie groß ist der Anteil der Beschäftigten und der Arbeitgeber, für die tarifliche Regelungen bestehen?

Um empirisch ausreichende, detaillierte und verlässliche Daten und Einschätzungen zu den Fragen zu bekommen, wurde ein Methodenmix gewählt: Zur Erfassung der wirtschaftlichen und be-
Zur Validierung der Erkenntnisse wurden erste Fassungen des Abschlussberichts dieser Studie mit fünf Vertretern und Experten der Arbeitgeberlandschaft reflektiert und diskutiert, was zu Ergänzungen und Korrekturen führte.

\section{Fünf zentrale Ergebnisse}

Die Zukunftsbranche Sozialwirtschaft, so die generelle Einschätzung der Studie, droht sich durch zersplitterte Repräsentations- und Verhandlungsstrukturen selbst auszubremsen.

\section{"Attraktive Arbeitsbedingungen in der Sozialwirtschaft hängen davon $a b$, wie viele öffentliche und halböffentliche Mittel zur Verfügung stehen"}

schäftigungsmäßigen Bedeutung der Sozialwirtschaft wurde auf die amtliche Statistik zugrückgegriffen, v. a. auf die Beschäftigtenstatistik der Bundesagentur für Arbeit sowie auf die Umsatzsteuerstatistik, die Pflegestatistik und auf die Kinder- und Jugendhilfestatistik.

Zur Erfassung der Tarifverträge und ihrer Regelungsgegenstände wurden zum einen einschlägige Publikationen - etwa vom Wirtschafts- und Sozialwissenschaftlichen Institut (WSI) - gesichtet. Zum anderen wurde auf das Tarifregister von ver.di - Vereinte Dienstleistungsgewerkschaft zurückgegriffen. Im Dialog mit den Tarifregisterexperten und den zuständigen Mitarbeiterinnen und Mitarbeitern bei den Gewerkschaften werden darüber hinaus ergänzende Informationen zu dem Anteil derjenigen Betriebe erfragt, die von den tariflichen Regelungen abgedeckt werden.

Die skizzierten Arbeitsschritte wurden ergänzt durch vertiefende Leitfadeninterviews mit Experten aus der Sozialwirtschaft. Sie dienten dazu, detaillierte Informationen zur Struktur, Organisation und Inhalten des sozialen Dialogs insbesondere aus Arbeitgebersicht zu erhalten. Des Weiteren war es Ziel der Interviews, Informationen zu zentralen Kooperationshemmnissen, deren Ursachen und zu zukünftigen Gestaltungsherausforderungen des sozialen Dialogs in der Sozialwirtschaft zu bekommen.
1 Sozialwirtschaft ist Zukunftsbran- che: Die Sozialwirtschaft hat für Gesellschaft, Politik und Wirtschaft eine Schlüsselbedeutung. Ihre Leistungen sind Zukunftsvorsorge, Sicherheit, Lebensqualität und soziale Gerechtigkeit. Gleichzeitig ist die Sozialwirtschaft mit derzeit circa zwei Millionen sozialversicherungspflichtigen Beschäftigten und knapp 300.000 geringfügigen Beschäftigungsverhältnissen einer der größten Beschäftigungsträger und eine große Schubkraft für mehr Arbeitsplätze und für nachhaltiges Wachstum. Prognosen rechnen in den kommenden zwanzig Jahren mit einem Zuwachs von mehr als 600.000 Arbeitsplätzen - der größte Teil davon in der Altenhilfe.

2. Sozialwirtschaft ist heterogen: Die Anbieterlandschaft in den sozialen Dienstleistungen ist sehr heterogen. Ein besonderes Gewicht haben freigemeinnützige Anbieter, die unter dem Dach von insgesamt sechs Wohlfahrtsverbänden zusammenarbeiten. Hinzu kommen noch öffentliche, zumeist kommunale, sowie private Dienstleister. Letztere haben in den letzten Dekaden insbesondere in der Altenhilfe an Bedeutung gewonnen. Die Sozialwirtschaft in Deutschland ist mithin durch einen Wohlfahrtsmix und einen Trägerpluralismus gekennzeichnet. Auch die Finanzierungsgrundlagen für die gebotenen Leistungen sind heterogen, allerdings dominieren öffentliche und halböffentliche (Stichwort Sozialversicherungen) Quellen. Die Knappheit der Mittel ist vielfach für wirtschaftliche Sorgen bei Einrichtungen und Unternehmen der Sozialwirtschaft verantwortlich und stellt auch aus Sicht der Bevölkerung die Frage nach einer zukunftsfähigen Gestaltung der Sozialwirtschaft.

3. Achillesferse Arbeit: Um die Löh- ne und Arbeitsbedingungen in der Sozialwirtschaft ist es in Deutschland schlecht bestellt. Große Teile der Branche liegen unterhalb des gesamtwirtschaftlichen Durchschnitts. Insbesondere in den stark wachsenden Teilen der Sozialwirtschaft - vor allem in der Altenhilfe - schlägt sich dies in wachsenden Schwierigkeiten bei der Rekrutierung von Arbeitskräften nieder. Mittelund langfristig ist mit einer drastischen Verschärfung der Probleme zu rechnen, $\mathrm{da}$ auch andere Wirtschaftsbranchen, in denen teilweise unter deutlich besseren Arbeitsbedingungen gearbeitet wird, um die aus demografischen Gründen knapper werdenden Arbeitskräfte konkurrieren. Eine weitsichtige und nachhaltige Arbeits- und Tarifpolitik ist hier gefordert, bessere Lohn- und Arbeitsbedingungen zu schaffen, um die Branche zukunftsfähig zu gestalten. Andernfalls droht die Zukunftsfähigkeit der Sozialwirtschaft an der Arbeit zu scheitern. $\mathrm{Ob}$ in der Sozialwirtschaft attraktive Arbeitsbedingungen entstehen können, das hängt zum einen davon ab, wie viele öffentliche und halböffentliche Mittel für diese Branche zur Verfügung stehen. Zum anderen ist aber auch relevant, ob sich die Arbeitgeber untereinander und mit den Interessenvertretungen der Arbeitnehmer auf entsprechende Perspektiven verständigen können.

4. Arbeitgeber-Arbeitnehmer-Beziehungen zersplittert: Die Vielfalt der Anbieterlandschaft in der Sozialwirtschaft spiegelt sich auch in den Arbeitgeber-Arbeitnehmer-Beziehungen und im System der Verhandlungen und Entscheidungen über Einkommens- und Arbeitsbedingungen wider. In "normalen « deutschen Wirtschaftsbranchen verhandelt eine Gewerkschaft mit einem Arbeitgeberverband über Bedingungen, die mehr oder weniger branchenweit gelten. In der Sozialwirtschaft wird demgegenüber in acht unterschiedlichen Arenen verhandelt und entschieden (vgl. Wohlfahrtsverbän- 
de). Zusätzlich kennt die Welt der Arbeitgeber-Arbeitnehmer-Beziehungen in der Sozialwirtschaft auch noch zwei Rechtssysteme: Für die kirchlichen Träger gilt ein eigenes, im Kirchenrecht verankertes Arbeits- und Mitarbeitervertretungsrecht. Diese außergewöhnliche Vielfalt und Zersplittertheit findet ihren Niederschlag in einer unübersichtlichen, ja intransparenten Heterogenität von rund 1.500 Tarifabschlüssen und arbeitsrechtlichen Vereinbarungen, von denen - mit wachsender Tendenz - fast zwei Drittel auf der betrieblichen Ebene, also dezentral, gefunden werden. Diakonie (11 Arbeitsrechtliche Kommissionen) und Caritas (6 Regionalkommissionen) gehen von einer hohen Flächendeckung ihrer Tarifaktivitäten aus. Die Untersuchung hat gezeigt, dass in der Sozialwirtschaft die Tarifabschlüsse oft als Notlagenabschlüsse realisiert werden, um mit verschlechterten Einkommens- und Arbeitsbedingungen auf wirtschaftliche Probleme des jeweiligen Unternehmens oder der jeweiligen Einrichtung reagieren zu können.

5. Befund Sociosclerose - System der arbeitspolitischen Interessenorganisation dämpft Zukunftsfähigkeit: Die außergewöhnlich vielfältige Struktur des Systems der Arbeitgeber-ArbeitnehmerBeziehungen in der deutschen Sozialwirtschaft ist eine Gefährdung der $\mathrm{Zu}$ kunftsfähigkeit dieser Branche. In vielen deutschen Wirtschaftsbranchen sorgen einheitliche und branchenweite Repräsentations- und Verhandlungsstrukturen dafür, dass es halbwegs einheitliche Lohn- und Arbeitsbedingungen gibt und der Wettbewerb unter den Anbietern über Preise oder Qualität der Produkte und Dienstleistungen erfolgt. Die Vielfalt und Dezentralität der Repräsentationsund Verhandlungsstrukturen in der Sozialwirtschaft ermuntert jedoch in der Branche, genau in die andere Richtungen zu denken und zu handeln: Unternehmen und Einrichtungen, die in wirtschaftlichen Schwierigkeiten sind, können versuchen, ihre Probleme durch Lohnabschlüsse und Arbeitsbedingungen unterhalb des bislang Branchenüblichen zu überwinden. In einer Branche, in der viele Anbieter nicht zuletzt aufgrund der knappen öffentlichen und halböffentlichen Mittel - mit wirtschaftlichen Schwierigkeiten zu kämpfen haben, entsteht so bei den Einkommens- und Arbeitsbedingungen die Gefahr eines Sogs nach unten. Dies erschwert es der Sozialwirtschaft außerordentlich, mittel- und langfristig für Arbeitnehmerinnen und Arbeitnehmer eine attraktive Branche zu werden - was angesichts der gewandelten Bedingungen am Arbeitsmarkt aber unerlässlich ist. Und zudem erschwert die Vielfalt des Repräsentations- und Verhandlungssystems, dass die Interessen der Gesamtbranche gegenüber der Öffentlichkeit, der Politik und gegenüber anderen Branchen offensiv und einheitlich vertreten werden können.

Die beschriebenen Institutionen der sozial- und arbeitspolitischen Interessenvertretung, Verhandlungen und Entscheidungen hindern die Sozialwirtschaft mithin daran, ihre Probleme in der Welt der Arbeit zu kurieren und dämpfen damit die Zukunftsfähigkeit der Branche. Unser Befund: Die Sozialwirtschaft droht sich auf der Suche nach ihrer Zukunftsfähigkeit in ihren arbeitspolitischen Institutionen zu verheddern; die Sozialwirtschaft leidet unter »Sociosclerose«.

\section{Interpretation der Ergebnisse: Neuaufstellung sinnvoll!}

Der Befund "Sociosclerose" wurde inspiriert durch die politikwissenschaftliche Denktraditionen zur »Institutionellen Scleroses " (Olson 1985) und in Anlehnung an die Debatten in Europa in den 1970er und 1980er Jahren über »Eurosclerose« gewählt.

Die europäische Wirtschaft stagnierte, weil sie sich in ihrer Zukunftsfähigkeit in einem Gestrüpp von miteinander nicht kompatiblen Regulierungen verfangen hatte. Die »Eurosclerose« wurde 1986 mit der Einheitlichen Europäischen Akte überwunden. Dabei handelte es sich um einen bewussten, von weitsichtigen Politikern geplanten Schritt zur Verbeiterung und Vertiefung der Europäischen Integration.

Werden die Verantwortlichen in der deutschen Sozialwirtschaft zu vergleichbar mutigen Schritten in der Lage sein? Der Weg dahin kann nur durch den Aufund Ausbau eines organisierten sozialen Dialogs, einer von den organisierten Sozialparteien gemeinsam getragenen »dialogisch-sozialpartnerschaftlichen Modernisierung « geebnet werden.

Impulse aus einem Europäischen Sozialen Dialog können für eine solche institutionelle Modernisierung Orientierung und Ermutigung sein. In Österreich und in den Niederlanden gelang in den letzten 15 Jahren eine grundsätzliche Neuaufstel- lung, weg von der Zersplitterung und hin zu branchenweit wirkenden Repräsentations- und Verhandlungsstrukturen.

Begünstigend für eine entsprechende Entwicklung in Deutschland könnte wirken, wenn es der Gewerkschaft ver.di sowie den einschlägigen Berufsverbänden gelänge, ihre Interessenvertretungsstrategien besser zu harmonisieren. Und hilfreich könnte auch sein, wenn die einschlägige Sozialwirtschaftsforschung ihre Aufmerksamkeit für die Bedeutung der institutionelle Grundlagen der arbeitspolitischen Entscheidungsfindung in der Sozialwirtschaft ausbauen würde - und dabei offensiv den gestaltungsorientierten Dialog mit Verantwortlichen aus Wirtschaft, Arbeitnehmerinteressenorganisationen und Politik sucht.

\section{Literatur}

Die Gesamtauswertung des Projekts »Promoting Employers' Social Services Organisations in Social Dialogue über alle 11 Länder hinweg wurde von Jane Lethbridge (2012) veröffentlicht; die deutsche Länderstudie von Evans u. a. (2012a) publiziert. Eine kondensierte Fassung erschien als Evans u. a. 2012b in: Theorie und Praxis der Sozialen Arbeit, Nr. 5, S. 369-377.

Dijk, van M./Essen, van G. (2012): Social dialogue in caring for the disabled, the elderly and in child care: The state of affairs in the Netherlands, The Netherlands country report to the European Project "Promoting employers' social services organisations in social dialogue«, Utrecht. Evans, M./Galtschenko, W./Hilbert, J. (2012a): Befund "Sociosclerose«: Arbeitgeber-Arbeitnehmer-beziehungen in der Sozialwirtschaft in Deutschland in Modernisierungsverantwortung. German country report to the European Project »Promoting employers' social services organisations in social dialogue», Gelsenkirchen. Evans, M./Galtschenko, W./Hilbert, J./Steinke, J. (2012b): "Sociosclerose«: Arbeitgeber-Arbeitsnehmerbeziehungen in der Sozialwirtschaft wenig zukunftsfähig. In: Theorie und Praxis der Sozialen Arbeit, Nr. 5, S. 369-377.

Jane Lethbridge, J. 2012: PESSIS - Promoting employers' social services in social dialogue, Final European Report, London: University of Greenwich. Olson, M. 1985: Aufstieg und Niedergang von Nationen: ökonomisches Wachstum, Stagflation und soziale Starrheit, Tübingen: Mohr.

Perl, Christian 2012: Social Dialogue in the Health and Social Service Sector, Austrian country report to the European Project »Promoting employers' social services organisations in social dialogue«, Vienna. 